\title{
A simple and efficient method for the preparation of live leukocytes from peripheral blood using the LeukoCatch ${ }^{\mathrm{TM}}$ system
}

\author{
Ayumi Okamoto ${ }^{1 *}$, Kosuke Torigata $^{1 *}$, Minami A. Sakurai ${ }^{1}$, Daisuke Okuzaki ${ }^{1,2}$, Hodaka Fujii ${ }^{3}$, \\ Toshinari Ohmine ${ }^{4}$, Daisaku Miura ${ }^{5}$, Shoichi Kimura ${ }^{6}$, Norikazu Yabuta ${ }^{1}$, Hiroshi Nojima ${ }^{1,2 \#}$ \\ ${ }^{1}$ Department of Molecular Genetics, Osaka University, Osaka, Japan \\ ${ }^{2}$ DNA-Chip Development Center for Infectious Diseases, Osaka University, Osaka, Japan \\ ${ }^{3}$ Combined Program on Microbiology and Immunology, Research Institute for Microbial Diseases, Osaka University, Osaka, Japan \\ ${ }^{4}$ Laboratory for Clinical Investigation, Osaka University Hospital, Osaka University, Osaka, Japan \\ ${ }^{5}$ Department of Pharmacy, School of Pharmacy, Hyogo University of Health Sciences, Kobe, Japan \\ ${ }^{6}$ Fukae Kasei Co., Ltd., Kobe, Japan \\ Email: ${ }^{\text {snj-0212@biken.osaka-u.ac.jp }}$
}

Received 16 June 2012; revised 27 July 2012; accepted 14 August 2012

\begin{abstract}
Leukocytes from peripheral blood (PB) are of great value for diagnosis as well as basic and clinical research. However, no easy, centrifugation-free method is available for the isolation of live leukocytes from blood. We here develop a simple and quick method for the purification of viable leukocytes from whole blood using novel tools, named tLeukoCatch (tip-type) or sLeukoCatch (syringe-type), which is equipped with three Pall filter layers and captures leukocytes but not red blood cells (RBCs) in whole blood. Indeed, we showed that several million leukocytes per $\mathrm{mL}$ ( $\sim 35 \%$ of the recovery rate) were captured and eluted from whole blood. The number of contaminant RBCs decreased from several million to several thousand. When mouse blood was hemolysed, almost all of the lysed RBC fragments were removed by passage through sLeukoCatch. Optical microscopic observation confirmed that the recovered leukocytes were sufficiently healthy to respond to growth stimuli. Efficient leukocyte recovery was also confirmed for hemolysed human blood. These results suggest that the LeukoCatch ${ }^{\mathrm{TM}}$ system is useful for bedside diagnosis and basic research with blood samples.
\end{abstract}

Keywords: Novel Tool; Leukocyte Purification; Centrifugation-Free; Diagnosis; PBMC

\section{INTRODUCTION}

Leukocytes play important roles in maintaining the de-

\footnotetext{
${ }^{*}$ These two authors contributed equally to this work

${ }^{\#}$ Corresponding author.
}

fense system of the body by regulating inflammatory processes and initial responses to microbial infections. Peripheral blood (PB) mononuclear cells (PBMCs) or peripheral blood leukocytes (PBLs) is ideal cells for diagnosis as well as basic and clinical research. Cultured leukocytes from patients have been useful materials in diagnostic and therapeutic applications for various autoimmune diseases and cancers. Indeed, the characterization of PBLs from animal disease models and patients has aided in the elucidation of the pathological mechanisms of rheumatoid arthritis and other autoimmune disorders [1-3].

With the rapid development of immunology in the past decade, many new immunotherapeutic strategies have been proposed. Immunotherapy based on antitumor immune memory is a new modality for cancer treatment that holds great promise for improving patient survival with minimal toxicity. Other cancer therapies that have drawn recent interest include cancer vaccination, which improves the antitumor activity of $\mathrm{T}$ cells from cancer patients; cell transfer therapy, which uses chimeric antigen receptors to activate human $\mathrm{T}$ cells to attack tumor cells; and the stimulation of other leukocyte types $[4,5]$. The isolation and characterization of PBLs and circulating tumor cells (CTCs) also could contribute to the early detection of cancer, selection of effective treatment, and estimation of prognosis [6]. Accordingly, effective techniques for handling PBLs and CTCs are needed.

Leukocytes can be separated from whole blood by rapidly sedimenting the red blood cells (RBCs) by centrifugation. The resulting supernatant contains mainly leukocytes, platelets, and a few RBCs. The most common and classical method for this process, known as Ficoll 400 (or Percoll) gradient centrifugation, is based 
on methodology established through the pioneering work of Bøyum [7]. A routine one-step centrifugation procedure splits the blood cells into two major fractions: after centrifugation, mononuclear cells are located on the top of the separation fluid, whereas erythrocytes and granulocytes have sedimented to the bottom [8].

Modifications of the density gradient centrifugation method and medium formulation have improved the quality of isolation and analysis of highly purified leukocytes from PB [9].

Some of these techniques are now available as commercialized kits, such as Ficoll-Hypaque [10]. Nevertheless, these leukocyte separation procedures require laboratory-based professional and time-consuming procedures using instrumentation that is not routinely available at the bedside or in a common clinical facility. Moreover, their protocols are too complex to apply to an automated machine to treat hundreds of samples simultaneously.

Novel methods to replace the gradient centrifugation method have been reported, which are based on immunomagnetic separation techniques. These methods are characterized by their specificity of targeting PBLs with a simple procedure $[11,12]$. However, they yield a biased population of leukocytes because only a limited number of leukocytes associate with the antibodies that are attached to the magnetic beads. Thus, a quick, easy, inexpensive, nonbiased, and efficient method for the purification of PBLs from RBC extracts is required to expand the utility of leukocytes as a diagnostic target.

Serum immunoglobulin and RBC hemoglobin comprise most of the protein in blood cells and must be removed before the protein components in leukocytes can be analyzed. New devices for trapping and removing PBLs, such as leukocytopheresis $[13,14]$, granulocytemonocyte-apheresis [15] and Adacolumn [16], have been developed and are used in clinical practice for the treatment of inflammatory bowel disease and rheumatoid arthritis [17]. A less expensive filtration device, called LeukoTrap (Pall, Leukosorb B Medium, LKB-3R), is available that efficiently captures leukocytes but not RBCs or platelets. This tool has been successfully used at the bedside [18] to prepare leukocyte-depleted platelet concentrates from whole blood [19-21]. These devices are mainly intended to remove PBLs and purify RBCs, and the captured PBLs are discarded.

By utilizing previous filtration systems (such as LeukoTrap) in an opposite manner, we assumed that we could obtain an efficient device to capture and recover sufficiently pure PBLs, with minimum contamination by RBCs. We present a novel technique, called the tLeukoCatch (tip-type) or sLeukoCatch (syringe-type) system, which allows the one-step separation of PBLs from RBCs in blood samples. The basic design of this system is similar to that of our original LeukoCatch ${ }^{\mathrm{TM}}$ tool [22], which consisted of a layer of filters held at the bottom of a tip or a syringe that captured leukocytes but not RBCs from a blood sample. Notably, the recovered PBLs are viable and can be efficiently expanded in a culture dish; this feature may expand the use of PBLs as research and diagnostic targets. The results from the present study indicate that tLeukoCatch and sLeukoCatch provide a readily available, easily processed, and convenient source of functional human PBLs for use not only in basic research of the human immune system but also in clinical research for the diagnosis of PBL-related diseases.

\section{MATERIALS AND METHODS}

\subsection{Cell Culture}

Human prostate cancer PC-3 cells (CRL-1435: ATCC, Manassas, VA) and mouse prostate cancer TRAMP-C1 cells (CRL-2730: ATCC, Manassas, VA) were maintained in Dulbecco's modified Eagle's medium (DMEM, Sigma-Aldrich Co., Milwaukee, WI) with $5 \%$ or $10 \%$ fetal bovine serum (FBS, HyClone, Logan, UT) supplemented with $100 \mathrm{U} / \mathrm{mL}$ penicillin, $100 \mu \mathrm{g} / \mathrm{mL}$ streptomycin, 10\% Nu-Serum IV (BD Biosciences), and $10 \mathrm{nM}$ dihydrotestosterone (DHT) in a $\mathrm{CO}_{2}(5 \%)$ incubator at $37^{\circ} \mathrm{C}$. Cells in the logarithmic growth phase were used for experiments.

\subsection{Application of sLeukoCatch to PC-3 Samples}

PC-3 cells $\left(1.7 \times 10^{6}\right.$ cells/experiment $)$ suspended in 2 $\mathrm{mL}$ of medium were passed through (sucked-and-poured) the sLeukoCatch five times. The captured PC-3 cells were eluted forcefully each time by fresh medium for five times. The five fractions of eluted PC-3 cells (first to fifth eluted fractions) were assessed for their viability by the trypan blue exclusion test. An equal number $(9.0 \times$ $10^{4}$ cells $/ \mathrm{mL}$ ) of nontreated PC-3 cells and the first fraction of eluted PC-3 cells were seeded separately in each plate with DMEM. The cell growth rates over 6 days were measured by the trypan blue exclusion test.

\subsection{Flowcytometry}

Leukocytes $\left(2.0 \times 10^{6}\right.$ cells/experiment $)$ eluted from sLeukoCatch were stained by the CycleTEST ${ }^{\mathrm{TM}}$ PLUS DNA Reagent Kit (BD Biosciences) according to the manufacturer's instructions. Samples were divided into four tubes. Cells were counted four times for each sample to obtain the mean value with a FACS Calibur and CellQuest software. This experiment was independently repeated twice.

To examine the viability of mouse peripheral lymphocytes eluted from sLeukoCatch, eluates were stained with propidium iodide (PI) and analyzed by flowcytometry. 
To examine the function of eluted mouse peripheral lymphocytes, eluted cells were stimulated with $5 \mu \mathrm{g} / \mathrm{mL}$ anti-CD3 antibody (145-2C11, BD Biosciences), $5 \mu \mathrm{g} / \mathrm{mL}$ anti-CD28 antibody (Clone 37.51, BD Biosciences), and $5 \mathrm{ng} / \mathrm{mL}$ mouse IL-2 (R\&D Systems) for 24 hours. Cells were stained with fluorescein isothiocyanate-conjugated anti-CD3 and phycoerythrin-conjugated anti-CD69 (H1.2 F3, BD Biosciences) antibodies and analyzed with FACS Calibur. To confirm that there was no difference in size and granularity between nontreated diluted mouse peripheral blood (mPB) and the first fraction of mouse lymphocytes eluted from sLeukoCatch, both samples were stained with PI and analyzed with FACS Calibur.

\subsection{Application of sLeukoCatch to Hemolysed Mouse Blood}

To prevent blood clotting, a heparin-coated $1 \mathrm{~mL}$ syringe was prepared by passing $\sim 0.05 \mathrm{~mL}$ of heparin sodium (1000 U/mL Novo-Heparin; Mochida Pharmaceutical Co., Ltd., Tokyo, Japan) through a syringe several times. A mouse was sacrificed by the injection of pentobarbital sodium (Somnopentyl, Kyoritsu Seiyaku, Tokyo, Japan), and its blood $(0.5 \mathrm{~mL})$ was obtained with the heaprin-coated $1 \mathrm{~mL}$ syringe. This experiment was performed with the lowest degree of neuropsychological sensitivity, pain, suffering and distress, which have been ensured by the employment of approved methods of anaesthesia and animal handling and by the application of specific antipain drugs whenever required. The collected blood was transferred into a collection tube with the heparin-coated syringe and diluted with $1 \mathrm{~mL}$ of phosphate-buffered saline (PBS) without $\mathrm{Ca}^{2+} / \mathrm{Mg}^{2+}$ ions [PBS(-)]. Next, 3.5 $\mathrm{mL}$ of RBC lysing buffer (Sigma-Aldrich Co., Milwaukee, WI) was added to the tube, for a total volume of 5 $\mathrm{mL}$. The blood mixture was drawn into a $10 \mathrm{~mL}$ sLeukoCatch syringe. To encourage hemolysis, the tube was gently turned upside-down by hand once per second for 5 minutes.

Lysed RBC ghosts were evacuated through gentle and slow movement (over 90 seconds) of the syringe piston. It was anticipated that most of the leukocytes would be captured by the filters during this process. Finally, $1 \mathrm{~mL}$ of PBS(-) or RPMI $+10 \%$ FBS was sucked into the sLeukoCatch syringe, incubated for 5 seconds, and then forcefully flushed back out into a plastic collection tube for subsequent analysis.

\subsection{Application of sLeukoCatch to Human Blood Samples without Hemolysis}

Blood samples (2 $\mathrm{mL}$ per tube) drawn from one of the authors (H.N, male, age 60) into vacuum blood collection tubes containing $1 \mathrm{mM}$ EDTA were sucked into the sLeukoCatch and poured back into the tube. After re- peating this process 5 times, supplemented DMEM (2 $\mathrm{mL}$ ) in a fresh container was sucked into the sLeukoCatch and poured back into the container to evacuate the captured cells. This process was repeated various times. The collected cells were incubated in $5 \% \mathrm{CO}_{2}$ at $37^{\circ} \mathrm{C}$.

\subsection{Application of sLeukoCatch to Hemolysed Human Blood Samples}

Blood samples collected from one of the authors (H.N, male, age 60) into heparin vacuum blood collection tubes ( $5 \mathrm{~mL}$ per tube) were passed through the sLeukoCatch syringe 5 times. Then, $5 \mathrm{~mL}$ of RBC lysing buffer in a 15 $\mathrm{mL}$ plastic tube was sucked into the sLeukoCatch syringe to induce hemolysis. The tubes were gently turned upside-down by hand once per second for 5 minutes at room temperature. The hemolysed sample was gently flushed into the same tube through a slow movement of the syringe piston over 2 minutes. Subsequently, the captured leukocytes were rinsed once with $5 \mathrm{~mL}$ of PBS(-) to remove the RBC ghosts. Fresh culture medium $(1 \mathrm{~mL})$ in a $15 \mathrm{~mL}$ plastic tube was sucked into the sLeukoCatch syringe. The captured leukocytes were eluted out into the same tube by strongly pushing the syringe piston.

\subsection{Hematologic Analysis of Blood Samples}

The numbers of leukocytes and RBCs were counted with an LH 750 hematology analyzer (Beckman Coulter, Fullerton, CA) immediately after the fresh blood sample was hemolysed and treated with or without the sLeukoCatch. Triplicate samples were counted.

\subsection{Ethical Permission}

All animal experiments were approved by the animal care and use committee of the Research Institute for Microbial Diseases, Osaka University (\#BikenA-H19-36-0). All experiments respected the welfare of animals and have been conducted strictly according to the legal and ethical requirements demanded by law. Details of animal welfare and steps taken to ameliorate suffering are included in the methods section of the manuscript.

All of the experiments utilizing human blood samples were performed after obtaining a written informed consent with the approval of the ethics committee of Osaka University (\#303).

\section{RESULTS}

\subsection{Structure and Use of the tLeukoCatch and sLeukoCatch Systems}

We previously reported that LeukoCatch ${ }^{\mathrm{TM}}$, equipped with five layers of filters (Pall, Leukosorb B Medium, LKB-3R), could be used to isolate whole protein extracts 
from leukocytes in human blood with minimum contamination by hemoglobin from RBCs [22]. We surmised that this system would also be useful for the preparation of live leukocytes with similar purity. For this purpose, we fabricated a tool containing three layers of Pall filters at the bottom of a plastic container, such as a pipette tip (tip-type; tLeukoCatch) or medical syringe (syringe-type; sLeukoCatch) (Figure 1A). We limited the number of filters to three because there was no difference in the efficiency of leukocyte capture when more than three filters were used in a similar LeukoCatch ${ }^{\mathrm{TM}}$ instrument [22]. Also, a three-filtered tool is easier to handle because blood movement through the filters is less sticky.

The handling process of tLeukoCatch or sLeukoCatch was simple and easy (Figure 1B). First, the lid of a vacuum collection tube ( $2 \mathrm{~mL}$ of blood per tube) was opened.
The blood sample inside was sucked up through the filters by moving the Pipetman's plunger button (for tLeukoCatch) or syringe piston (for sLeukoCatch) upward (Figure 1B(i); steps a and c) and downward (steps b and d) five times by hand. Then, the tip or syringe needle was immersed into a fresh container (e.g., a $15 \mathrm{~mL}$ plastic tube) containing supplemented DMEM, and $2 \mathrm{~mL}$ of the DMEM was sucked up into the upper-side space of the filter (Figure 1B(ii); step e) to wash the filters. The plunger button or piston was pushed down to evacuate the captured leukocytes into a fresh tissue culture plate (Figure 1B(ii); step f). This elution process may be repeated many times to increase the recovery rate of the captured leukocytes. The collected leukocytes may be stored frozen in the presence of $7 \%$ dimethyl sulfoxide (DMSO) or transferred to a tissue culture plate and maintained at $37^{\circ} \mathrm{C}$ in a $\mathrm{CO}_{2}(5 \%)$ incubator.

\section{A}

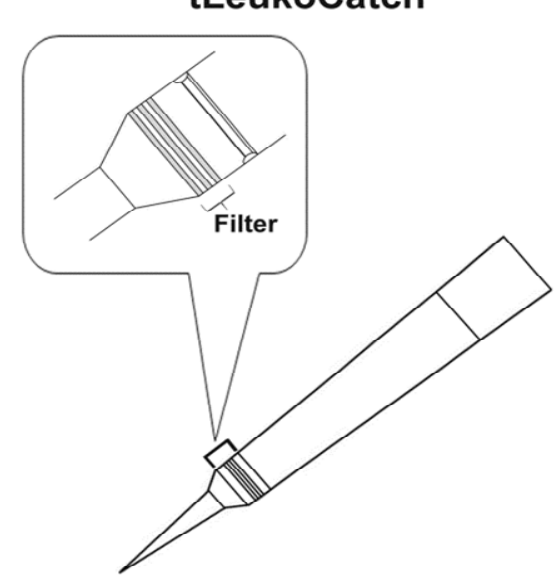

sLeukoCatch

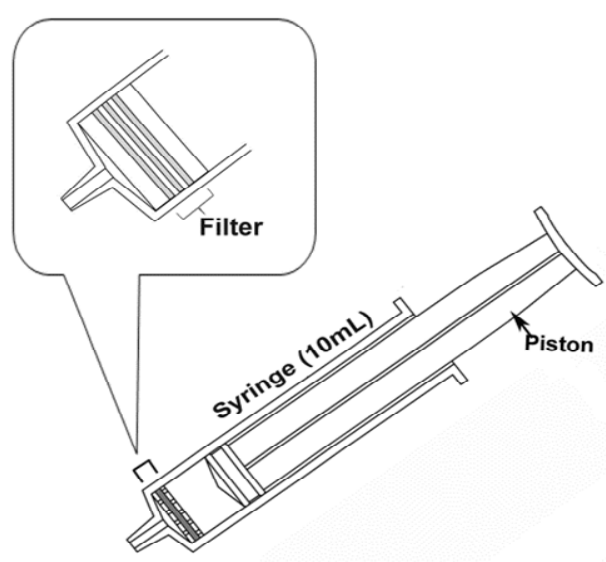

B

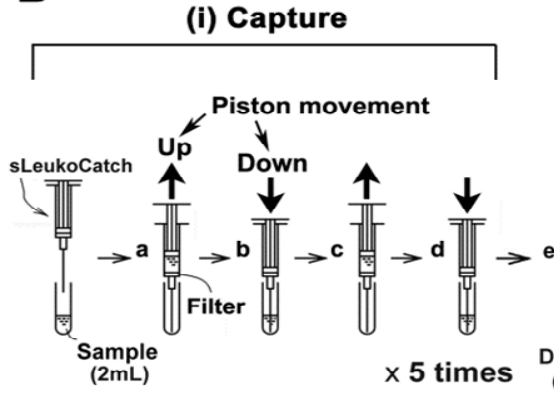

(ii) Elute
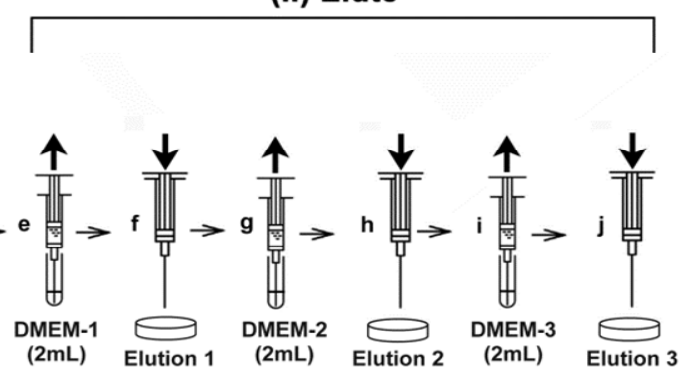

Figure 1. Schematic drawings of the LeukoCatch ${ }^{\mathrm{TM}}$ system and its usage. (A) Structure of the LeukoCatch ${ }^{\mathrm{TM}}$ system, in which the three-layered stacked filters are installed between the stoppers at the bottom of a syringe. An enlarged view of the stacked configuration of the filtering system is shown in an inset; (B) Schematic drawing of a protocol for the preparation of live leukocytes (or PBMCs) from a $2 \mathrm{~mL}$ blood sample. The protocol consists of two procedures: capture of leukocytes by the LeukoCatch ${ }^{\mathrm{TM}}$ filters (i) and leukocyte elution by a cell culture medium (e.g., DMEM) (ii). Thin horizontal arrows show the flow of the procedure (from step a to step $\mathrm{j}$ ). Thick vertical arrows indicate the multiple movements of the piston to allow the blood (i) or elution medium (ii) to pass through the stacked filters in the LeukoCatch ${ }^{\mathrm{TM}}$ system. In each step, the piston was moved upwards (steps a, c, e, g, and i) or downwards (steps b, d, f, h, and j) by hand. The whole procedure may be completed within 3 minutes. 


\section{2. sLeukoCatch Enabled the Capture and Recovery of Live Tissue Cultured Cells}

Before applying the LeukoCatch ${ }^{\mathrm{TM}}$ system to blood samples, we first tested its capture rate and recovery efficiency using tissue cultured cells. We tested the ability of the sLeukoCatch to collect human prostate cancer PC-3 cells. Briefly, PC-3 cells were passed through the filters by gently pushing and pulling the syringe piston five times. After passing the PC-3 cells through the sLeukoCatch five times, about $82 \%$ of PC-3 cells were captured (Figure 2A). PC-3 cells that were captured on the filters were eluted forcefully by passing the DMEM medium into a test tube ( $1-5$ times by serial passage). This procedure resulted in an elution recovery rate of $23 \%$ of captured PC-3 cells at the first passage (first fraction) (Figure 2B). The cells in the first eluted fraction were as healthy as nontreated PC-3 cells, as shown by their similar cell growth rates (Figure 2C) and images under a microscope (Figure 2D).

Next, we used the tLeukoCatch to collect mouse prostate cancer TRAMP-C1 cells using a Gilson Pipetman. The capture of TRAMP-C1 cells by tLeukoCatch was more efficient $(86 \%)$ than that by sLeukoCatch (Figure S1A); however, the elution rate $\left(11 \%\right.$ at $1^{\text {st }}$ elution) was much lower than that of sLeukoCatch even after the $5^{\text {th }}$ elution (Figure S1B), which can be probably attributed to the weak pipetting force of the Gilson Pipetman. Because tLeukoCatch was originally designed to treat large numbers of blood samples, each with a volume of less than $0.5 \mathrm{~mL}$ on an automated machine (see Discussion), we refrained from using tLeukoCatch for manual manipulations and used sLeukoCatch hereafter.

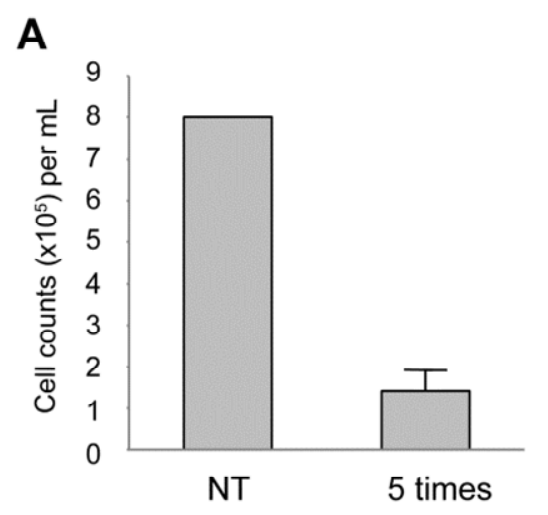

C

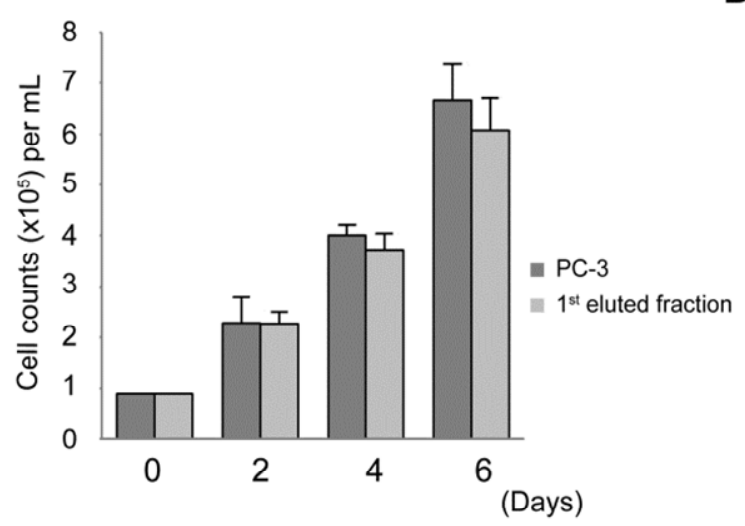

B

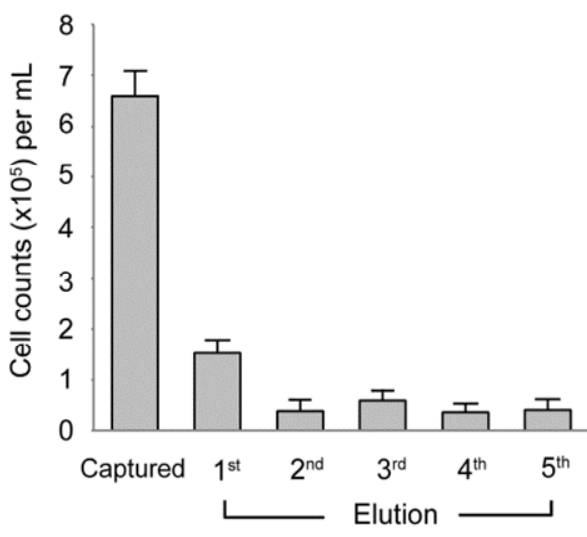

D

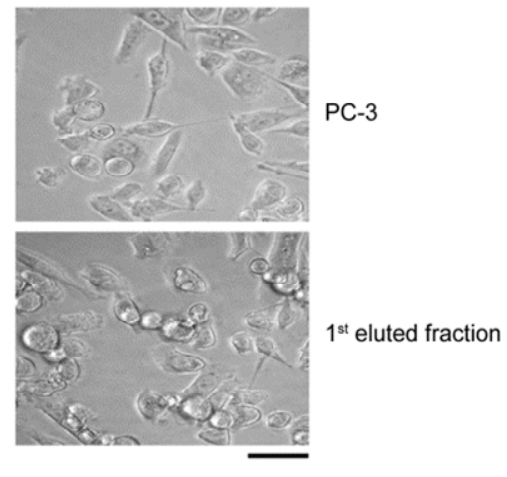

Figure 2. sLeukoCatch allow the capture and recovery of live PC-3 cells. (A) PC-3 cells were passed through an sLeukoCatch five times. NT, nontreated. Bar graphs represent the number of PC-3 cells (flow-through) that were not captured after being passed through the sLeukoCatch system five times; (B) PC-3 cells were passed through the sLeukoCatch system five times. Captured PC-3 cells were eluted by fresh medium (sLeukoCatch system); (C) Equal numbers of nontreated PC-3 cells and PC-3 cells from the first eluted fraction $\left(1^{\text {st }}\right)$ were seeded in separate plates with DMEM. Bar graphs represent the numbers of nontreated PC-3 cells and PC- 3 cells in the $1^{\text {st }}$ eluted fraction that were assessed by the trypan blue exclusion test over 6 days. Each data point represents an average of three independent experiments; (D) Cell images of nontreated PC-3 cells and PC-3 cells in first eluted fraction $\left(1^{\text {st }}\right)$ on day 4 . Bar $=50 \mu \mathrm{m}$. 


\section{3. sLeukoCatch Allows the Capture of Leukocytes from Human Peripheral Blood}

To isolate viable cells from human peripheral blood (hPB) by sLeukoCatch, a blood sample $(2 \mathrm{~mL})$ in a vacuum blood collection tube containing $1 \mathrm{mM}$ EDTA was aspirated into the sLeukoCatch by hand and blown out gently five times to capture the leukocytes (see Figure 1B(i)). Cells that were captured on the filters were eluted forcefully into a test tube containing $2 \mathrm{~mL}$ of fresh DMEM and $10 \%$ inactivated FBS. This process was repeated five times to maximize the number of collected cells (Figure 1A(ii)). Because the movement of the syringe piston was smooth even when whole blood was used, we did not dilute the blood sample by PBS(-).

Nearly $35 \%$ of the leukocytes or white blood cells (WBCs) were captured in the filters of the sLeukoCatch, as judged by the difference between the values of the nontreated (NT) and pass through (PS) samples (Figure 3A). The captured leukocytes were eluted efficiently by successive elution steps (Figures 3A and B; see columns $1^{\text {st }}$ to $\left.5^{\text {th }}\right)$. Almost all of the leukocytes were eluted into the medium by repeating the three elution processes (Figure 3B). Because the fourth and fifth eluted samples contained very small numbers of residual leukocytes that were hard to detect precisely, the ratio of eluted leukocytes from the filters in Figure 3B shows a value of $>100 \%$. From a practical perspective, the fourth and fifth elution steps may be skipped in future uses of the technique.

Similar distributions of neutrophils, lymphocytes, and monocytes, as detected by a hematology analyzer, were observed during the nontreated and elution processes, which suggested the nonbiased capture of these leukocytes (Figure 3C). The proportion of platelets included in the first to third eluted fractions were largely reduced, which suggested an efficient separation of platelets from the eluted leukocytes (Figure 3D). The number of RBCs decreased by two orders of magnitude, from $5.2 \times 10^{6}$ cells (nontreated hPB) to 68,000 cells (third eluted fraction) in $1 \mu \mathrm{L}$ of hPB (Figure 3E). Thus, the decreasing number of RBCs in the eluted samples increased the ratio of leukocytes to RBCs (Figure 3F). Microscopic observation clearly showed the successful separation of RBCs from leukocytes in the eluted fraction (Figure 3G). Taken together, these results show that intact leukocytes in hPB samples can be captured and eluted without any osmotic stresses in a procedure that requires handling for only a few minutes.

\section{4. sLeukoCatch Provides RBC-Free Leukocytes When Used for Hemolysed Mouse Blood}

The hemolysis of RBCs can be a convenient process to remove RBCs efficiently. However, leukocytes are also hemolysed, although much less efficiently, when they are immersed in hemolysis buffer for a long time. Thus, it is important to determine the proper timing at which RBCs but not leukocytes are hemolysed. We prepared mouse peripheral blood (mPB) and assessed the timing of hemolysis using various ratios of blood to hemolysis buffer. A ratio of 1:9 yielded almost complete hemolysis, as judged by the clear blood solution obtained after gently turning the tube upside-down once per second by hand at room temperature for 5 minutes (data not shown).

After 5 minutes, when almost all of the RBCs were hemolysed, we pushed down on the sLeukoCatch syringe piston slowly over 90 seconds to promote the capture of leukocytes but not RBC ghosts by the sLeukoCatch filters (Figure 4A). This process removed the serum and hemoglobin. We sucked $1 \mathrm{~mL}$ of PBS(-) or RPMI $+10 \%$ FBS into the sLeukoCatch syringe, incubated it for 5 seconds, and then flushed the solution back out by forcefully pushing the syringe piston to retrieve the captured leukocytes. Almost all of the lysed RBC fragments were removed through this process.

We tested whether the eluted leukocytes were viable. As shown in Figure 4B, a sizable population of lymphocytes was easily detected. The viability of the eluted cell population was more than $95 \%$ and was comparable to that of the hemolysed (but not eluted) cell population. These results show that treatment with sLeukoCatch did not markedly affect viability. To assess the functionality of the eluted mouse lymphocytes, the eluted cells were stimulated with anti-CD3 antibody and anti-CD28 antibody plus recombinant mouse IL-2 for 24 hours. The stimulated lymphocytes became larger (Figure 4C), suggesting their activated phenotype. Moreover, CD3 (+) cells showed markedly increased expression of CD69, an activation marker of $\mathrm{T}$ cells (Figure 4D). These results show that the lymphocytes purified with sLeukoCatch were functional.

We tested whether there was a difference in size and granularity between nontreated $\mathrm{mPB}$ cells and cells in the eluted fraction. A sizable population of lymphocytes was easily detected in both samples by flowcytometry (Figure 4E). No change in the size and granularity distribution of the lymphocytes was found after using the LeukoCatch $^{\mathrm{TM}}$ system. These results suggest that the eluted fraction contained the whole population of leukocytes.

\section{5. sLeukoCatch Retrieves RBC-Free Leukocytes from Hemolysed Human Blood}

We applied the hemolysis technique to hPB samples. Human blood was more resistant to the hemolysis buffer than mouse blood when it was diluted as described above 

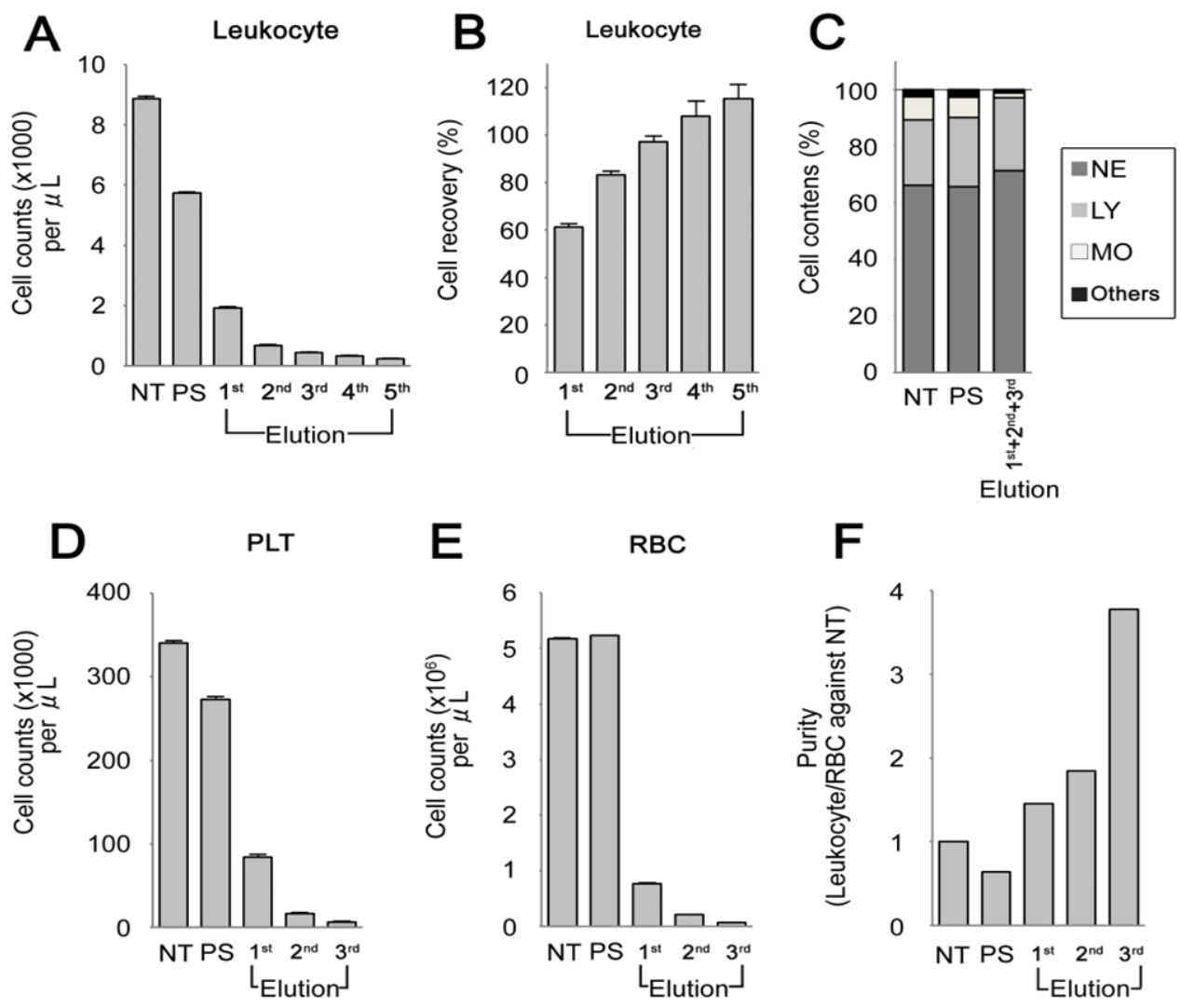

F

G

PS
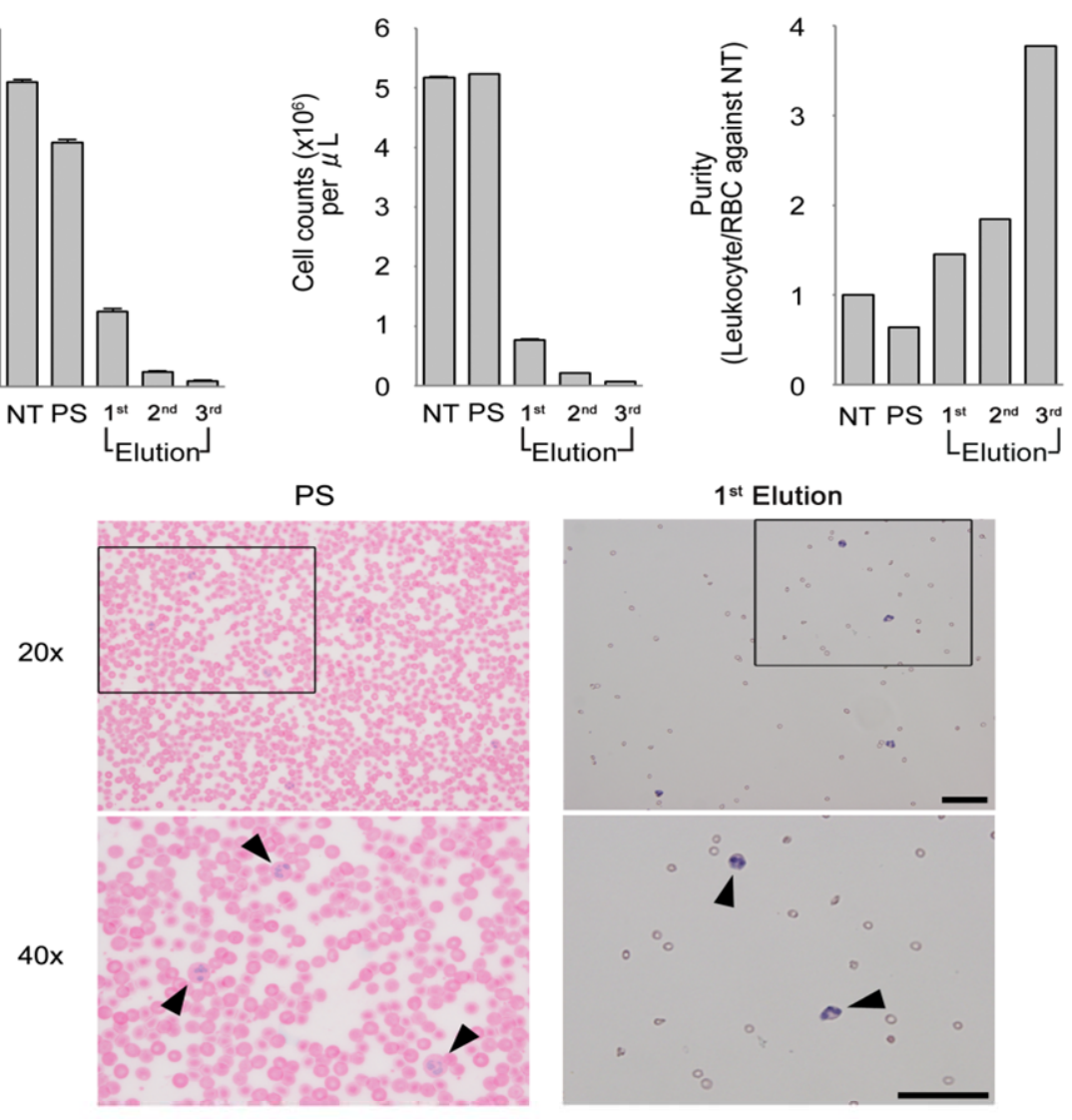

Figure 3. sLeukoCatch allows the efficient capture of RBC-free leukocytes from human peripheral blood (PB). (A) Numbers of leukocytes in the nontreated (NT), pass-through (PS), and first $\left(1^{\text {st }}\right)$ to fifth $\left(5^{\text {th }}\right)$ eluted fractions, respectively, are shown. Data were obtained with an LH1511 cell counter; (B) Ratio of eluted versus captured leukocytes before (NT) and after (PS and fractions $1^{\text {st }}$ to $5^{\text {th }}$ ) passage through the sLeukoCatch. Accumulated percentages of leukocytes eluted from sLeukoCatch at each elution step $\left(1^{\text {st }}\right.$ to $\left.5^{\text {th }}\right)$ are shown, revealing an almost complete recovery; (C) Percentage of neutrophils (NE), lymphocytes (LY), monocytes (MO), and others in the NT, PS, and eluted fractions $1^{\text {st }}+2^{\text {nd }}+3^{\text {rd }}$ after use of the sLeukoCatch; (D) and (E) Numbers of platelets (PLTs); (D) and red blood cells (RBCs); (E) in the PB, PS, or eluted fractions $1^{\text {st }}$ to $3^{\text {rd }}$ are shown. Cell numbers were counted with an LH1511 cell counter; (F) Relative RBC versus leukocyte content in the PB, PS, and eluted fractions $1^{\text {st }}$ to $3^{\text {rd }}$. Bar graphs for eluted fractions $4^{\text {th }}$ and $5^{\text {th }}$ are not shown because few RBCs were found; (G) Microscopic images of PS (left panels) and eluted fraction $1^{\text {st }}$ (right panels) at 20× and $40 \times$ magnifications. Regions displayed as enlarged images $(40 \times)$ are encircled in the $20 \times$ images. Arrowheads indicate putative leukocytes. Bar $=25 \mu \mathrm{m}$. 
A (i) Hemolysis
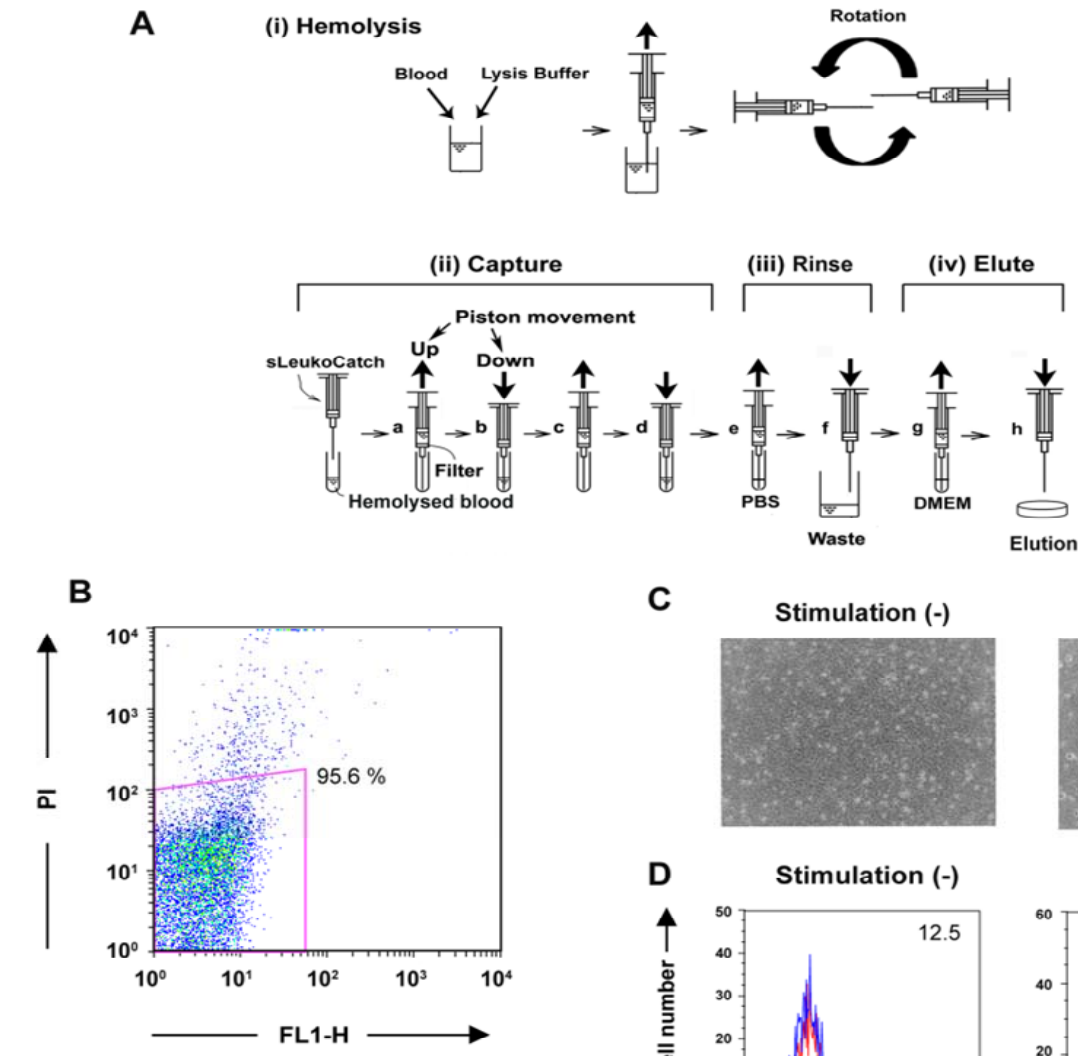

C

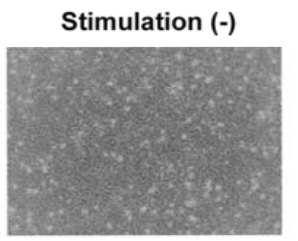

Stimulation (+)

D Stimulation (-)
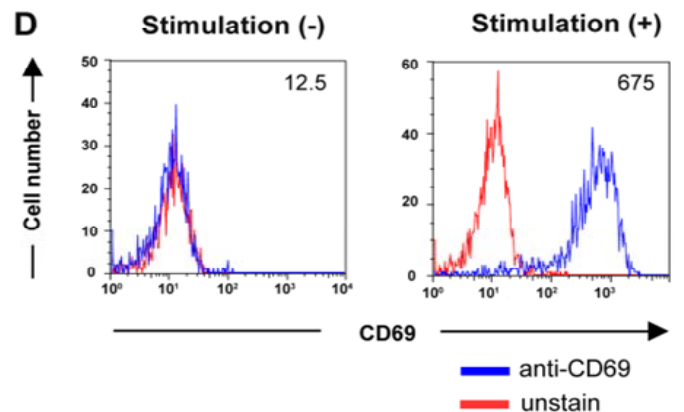

E

FSC
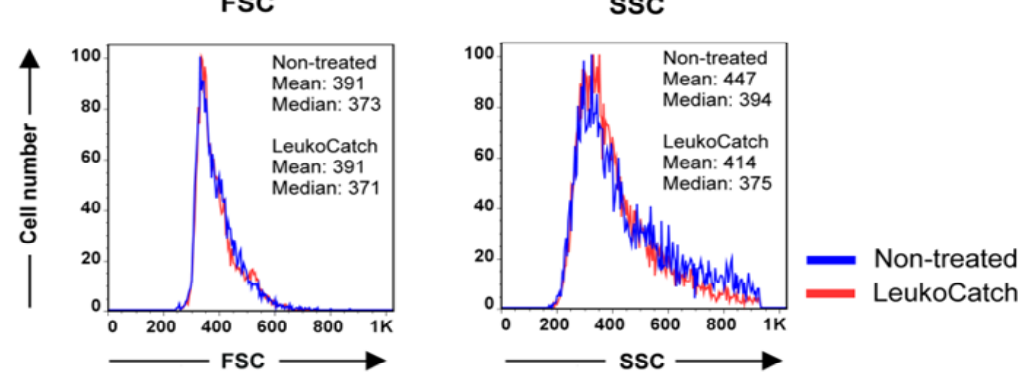

Figure 4. sLeukoCatch obtains live and functional leukocytes without RBC debris from hemolysed mouse peripheral blood (mPB). (A) Schematic protocol for the preparation of hemolysed mouse blood from $10 \mathrm{~mL}$ of diluted blood sample: (i) hemolysis of mouse blood, (ii) capture of leukocytes by the sLeukoCatch filters, (iii) rinse, and (iv) leukocyte elution by cell culture medium. Thin horizontal arrows show the flow of the process (from step a to h). Thick vertical arrows indicate the movements of the syringe piston to allow the blood (ii) or RPMI (iv) to pass through the stacked filters in the sLeukoCatch system. In each step, the piston was moved upwards (steps a, c, e, and g) or downwards (steps b, $\mathrm{d}, \mathrm{f}$, and h) by hand; (B) Flowcytometric analysis of an eluted sample. The eluted fraction from $\mathrm{mPB}$ was stained with propidium iodide (PI) and analyzed by flowcytometry, which identified leukocytes by their FSC and SSC properties as basic "lymphocyte gating"; (C) Microscopic images of eluted leukocytes after 24 hours with or without stimulation by anti-CD3 antibody, anti-CD28 antibody, and IL-2; (D) CD69 expression on nonstimulated or stimulated T cells was analyzed by flowcytometry. CD3 $(+)$ cells were gated for analysis of CD69 expression; (E) Histogram representing the lymphocyte size and granularity. Non-treated $\mathrm{mPB}$ and sLeukoCatch-mediated eluted fraction from $\mathrm{mPB}$ were analyzed by flowcytometry, which identified leukocytes by their FSC and SSC properties as lymphocyte gating. 
(data not shown). Thus, we performed hemolysis directly in the sLeukoCatch syringe (Figure 5A). Briefly, blood samples collected into heparin $(5 \mathrm{~mL}$ per tube) were passed through the sLeukoCatch syringe five times to remove most of the RBCs. Then, $5 \mathrm{~mL}$ of hemolysis buffer in a $15 \mathrm{~mL}$ plastic tube was sucked into the sLeukoCatch syringe. Hemolysis of the residual RBCs was induced by gently turning the tube upside-down by hand every second for 5 minutes at room temperature. The hemolysed blood sample was gently flushed into the same tube through a slow movement of a syringe piston over 90 seconds. Subsequently, the captured leukocytes were gently rinsed once by using $5 \mathrm{~mL}$ of PBS(-) to remove the RBC ghosts. Finally, fresh culture medium (1 $\mathrm{mL}$ ) in a $15 \mathrm{~mL}$ plastic tube was sucked into the sLeukoCatch syringe, and the captured leukocytes were eluted out into the same tube by forcefully pushing the syringe piston.

We found that the approximately $77 \%$ of the nontreated hPB sample $(\sim 6000 / \mu \mathrm{L})$ was in the PS fraction. The loss of leukocytes during the PBS(-) rinse process was small $(<17 \%)$, as judged by the hematologic analysis that was performed immediately after elution (Figure 5B). Notably, the captured leukocytes were almost completely recovered in the first and second eluted fractions (Figure 5B). Interestingly, the eluted leukocytes were almost free of RBCs and their ghosts, according to the hematologic analysis (Figure 5C) and microscopic observation (data not shown). Indeed, the ratio of leukocytes to RBCs increased markedly in the first $(76 \times)$ and second $(65 \times)$ eluted fractions (Figure 5D). These results indicate that sLeukoCatch retrieved about $10 \%$ RBC-free leukocytes in $2 \mathrm{~mL}$ from $5 \mathrm{~mL}$ of hemolysed $\mathrm{hPB}$. This number may be large enough to perform further analysis for diagnostics as well as basic and clinical research.

\section{DISCUSSION}

The successful identification of biomarkers for PBLs in infectious disease, autoimmunity, and cancer is critical not only for diagnosis but also for tracking drug effects at early time points in clinical trials, which may prevent costly late trial failures. Although popular, the Ficoll density centrifugation method is laborious and inaccurate. Therefore, a simple technique for the preparation of PBLs from whole blood has been eagerly awaited.

In the present study, we report a simple, rapid and efficient preparation technique called the LeukoCatch $^{\mathrm{TM}}$ system that enables the recovery of live leukocytes from mouse and human blood samples through blood handling for only a few minutes (Figure 1). Tissue cultured cells were effectively captured and recovered with this system, yielding healthy cells with a normal growth rate (Figure 2). When applied to human blood samples, sLeukoCatch effectively captured and successfully separated leuko- cytes from RBCs (Figure 3). A dramatic removal rate of $\mathrm{RBC}$ debris was observed in the recovered leukocytes from mouse blood samples when hemolysis was performed before elution (Figure 4). This procedure will be useful for samples in which hemolysis is not toxic to the required leukocytes.

We prepared two kinds of modified LeukoCatch ${ }^{\mathrm{TM}}$ systems: the tLeukoCatch and the sLeukoCatch. Because Gilson's Pipetman is manually handled, its evacuation ability is not strong enough to pass through all blood samples smoothly. Thus, the primary utility of the tLeukoCatch system is as an automated system, in which the evacuation power can be mechanically controlled, with large numbers of blood samples. In contrast, sLeukoCatch is useful when used for a small number of samples at the bedside. The tLeukoCatch and sLeukoCatch systems will contribute to the preparation of leukocytes or immune-related cells that can subsequently be analyzed by various means. It is particularly convenient for bedside use because it can be easily handled by those who are not familiar with medical sciences.

As one potential application, LeukoCatch ${ }^{\mathrm{TM}}$ may be helpful for improving the efficiency of cancer immunotherapies [4]. The basic strategy for tumor immunotherapy involves the stimulation of patient leukocytes, such as the cytotoxic T lymphocytes (CTLs), natural killer cells, or lymphokine-activated killer cells (LAKs), and their subsequent activation by putative tumor-specific antigens. Tumor-specific antigen peptides have been explored by mass spectrometry (MS) with clinical tumor samples, and identified peptide sequences have been registered in databanks after extensive research [23]. However, mainly due to economic reasons, only the topranking peptides in the list are applied to patients, without considering the differences in efficacy of the different peptides among individual tumors. The Leuko$\mathrm{Catch}^{\mathrm{TM}}$ system would enable clinicians to collect and isolate, in a simple and rapid manner at the bedside, live leukocytes and circulating malignant cells, which can subsequently be cultured, expanded, and applied to various detailed examinations.

We propose a novel strategy for tumor immunotherapy using tumor cells and leukocytes that are obtained freshly from each patient with the LeukoCatch ${ }^{\mathrm{TM}}$ system (Figure 6). This work flow consists of two parts: (A) the profiling and identification of tumor-specific peptides, and (B) the screening and evaluation of peptides for the stimulation of patient leukocytes. Both parts start from a culture step after use of the LeukoCatch ${ }^{\mathrm{TM}}$ system (Figures $6 A(i)$ and $B(i)$ ).

Tumor cells and leukocytes are cultured until a sufficient number of cells for analysis are obtained. The membrane fraction purified from tumor cells is applied to MS analysis to identify tumor-specific antigens. This 
A

(i) Capture

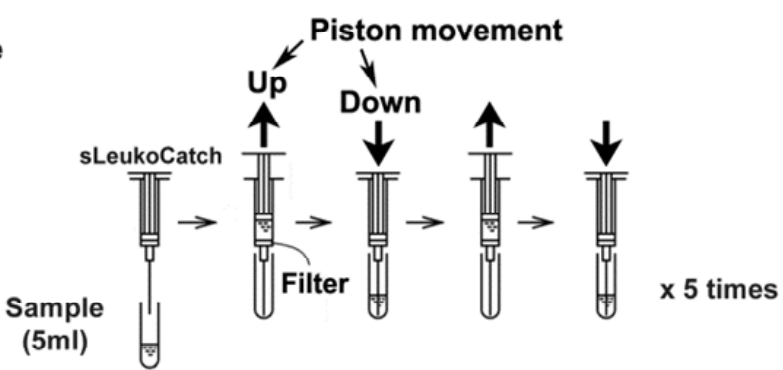

(ii) Hemolysis

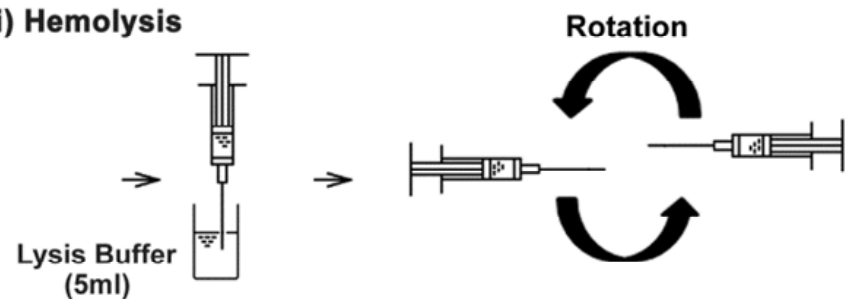

(iii) Rinse

(iv) Elute
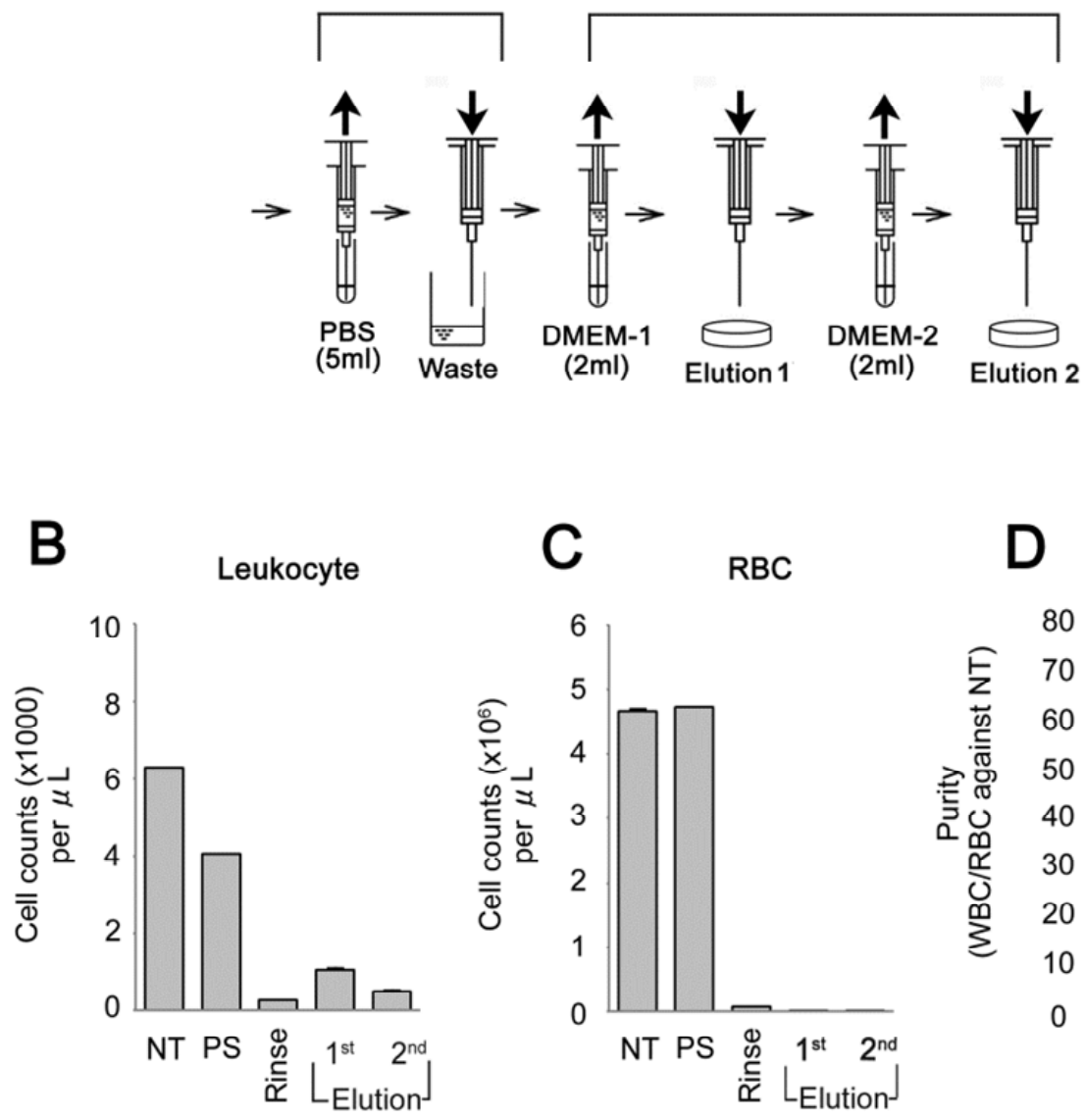

RBC

D
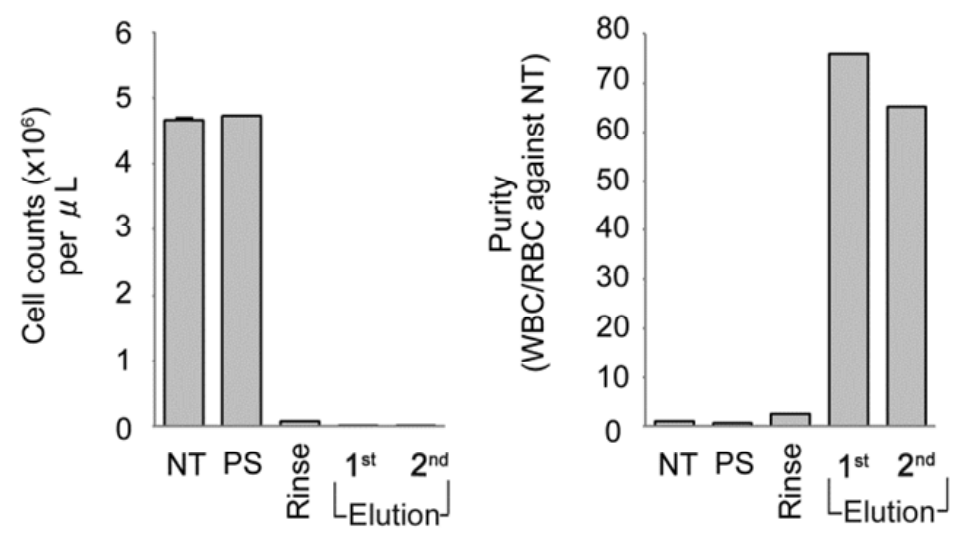

Figure 5. sLeukoCatch is useful for the isolation of RBC-free leukocytes from hemolysed human peripheral blood (hPB). (A) Schematic protocol for the purification of leukocytes from $5 \mathrm{~mL}$ of hemolysed hPB: (i) capture of leukocytes by the sLeukoCatch filters, (ii) hemolysis of captured cells, (iii) PBS rinse, and (iv) leukocyte elution by DMEM. Thin horizontal arrows show the flow of procedure (from step a to l). In each step, the syringe piston was moved upwards (steps a, c, e, g, i, and k) or downwards (steps b, d, h, j, and l) by hand; (B and C) Bar graphs indicate the number of leukocytes (B) or RBCs (C) in nontreated (NT), passed-through (PS), or eluted fractions $1^{\text {st }}$ and $2^{\text {nd }}$. Data were obtained with an LH1511 cell counter; (D) Bar graphs show relative amounts of leukocytes and RBCs that were included in the NT, PS, and eluted fractions $1^{\text {st }}$ and $2^{\text {nd }}$. 


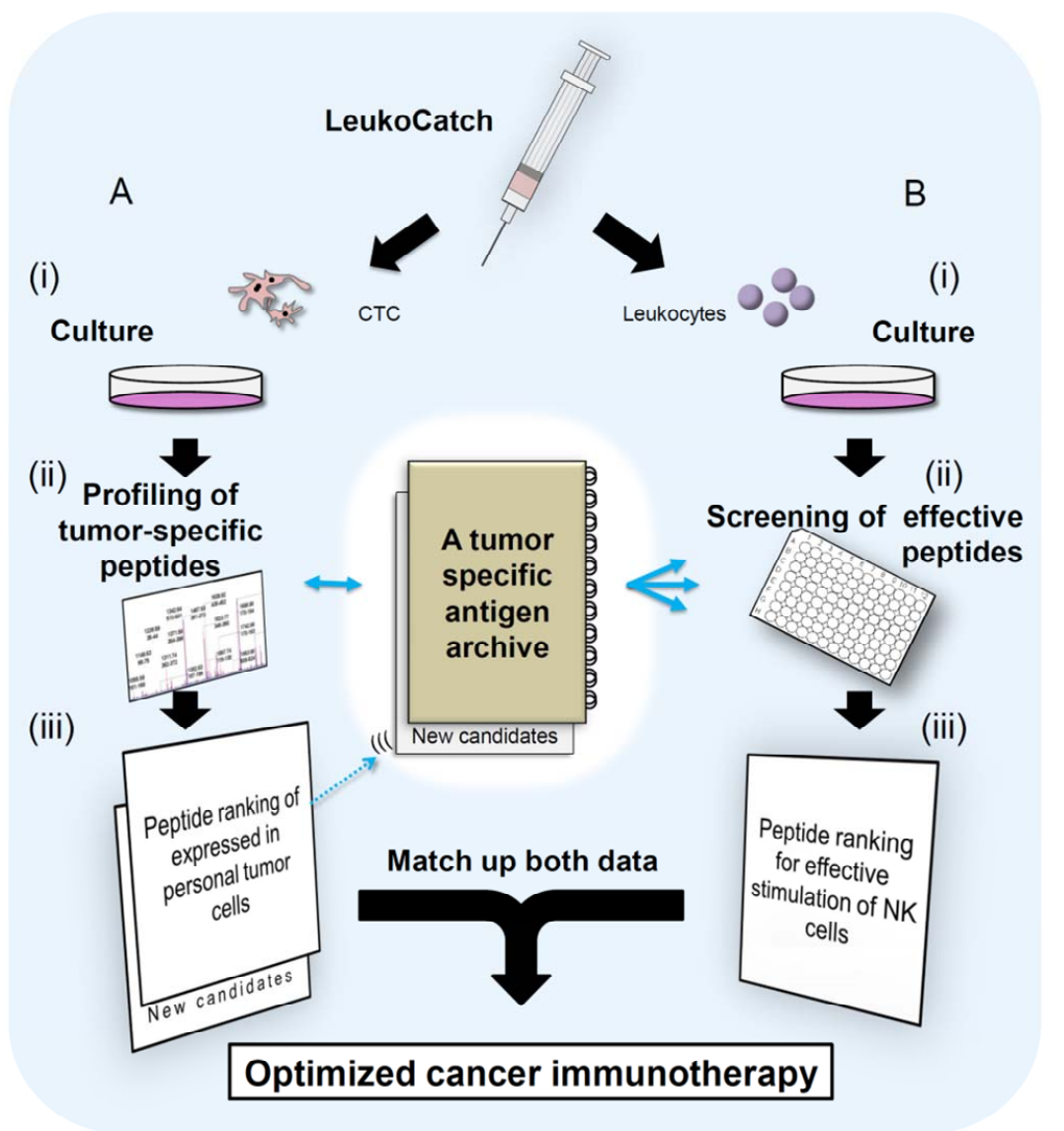

Figure 6. Schematic for the novel use of the LeukoCatch ${ }^{\mathrm{TM}}$ system for tumor immunotherapy. The LeukoCatch ${ }^{\mathrm{TM}}$ system enables the collection of CTCs and leukocytes simultaneously from PB samples at the bedside. (A) Collected tumor cells can be used for cell culture (i), antigen profiling (ii), and peptide ranking (iii) for the identification of suitable antigens from a preconstructed antigen library; (B) sLeukoCatch can also be used for cell culture (i), the screening of effective peptides (ii), and peptide ranking (iii) to pursue more effective stimulations by antigens. See the main text for details.

analysis is performed for several hundred patients to construct an archive of tumor-specific antigens. In this archive, the prevalent antigens for specific tumors are ranked according to their frequency of detection among tested patients (Figure 6A(ii)). The absence of proteins harboring the peptide sequences of these putative tumor-specific antigens in the membrane fractions of normal cells should be confirmed before the antigens are listed in the archive.

The existence of a preconstructed antigen archive plays an important role in this proposal because the peptide sequences obtained from the MS analysis of the patient tumor cells are screened against this archive to confirm their tumor specificity. Analysis of novel patients may lead to the identification of new candidate peptides, which may help to enrich the archive content (Figure 6A(ii)). For more effective therapy, in vitro stimulation of the patient leukocytes with these antigens may also be important (Figure 6B(ii)). In the final step, the most effective immunotherapeutic protocol is determined through the integration of the data (Figures 6A(iii) and $\mathbf{B}$ (iii)). Our findings showing that recovered PC-3 cells grow normally may support the practical applicability of this protocol at the bedside, which will be tested in our future works.

Taken together, the results of this study indicate that the LeukoCatch $^{\mathrm{TM}}$ system allows the rapid and simple preparation of leukocytes, which may be useful not only for bedside diagnosis but also for basic research using blood samples. This technique will contribute to the preparation of immune cells that can be reliably analyzed by various means.

\section{AUTHORS' CONTRIBUTIONS}

H.N. made contributions to the conception and design of this study and drafted the manuscript. S.K. constructed 
the LeukoCatch ${ }^{\mathrm{TM}}$ device. A.O., K.T. and D.M. performed the mouse study. A.O., K.T. and N.Y. contributed to the tissue culture experiments. T.O., A.O., M.A.S., D.O., and K.T. participated in the blood fractionation and data analysis. A.O., K.T. and H.F. contributed to the flowcytometry analysis of eluted leukocytes. All authors read and approved the final manuscript.

\section{FUNDING}

This work was supported in part by grants-in-aid for Scientific Research S (\#15101006), Scientific Research B (\#20370081) and Exploratory Research (\#1651085) from the Ministry of Education, Culture, Sports, Science and Technology of Japan (http://www.mext.go.jp/eng $\underline{\text { lish/) }}$ to HN., and Support Projects (\#H22-0910-KD77Go) from the New Energy and Industrial Technology Development Organization (NEDO), the Ministry of Economy, Trade and Industry of Japan to SK. No additional external funding was received for this study. The funders had no role in study design, data collection and analysis, decision to publish, or preparation of the manuscript.

\section{ACKNOWLEDGEMENTS}

We thank Ms. Kana Ao-Ooi of our laboratory for technical assistance and Mr. Shin Moto and Ms. Saki Terashita of Fukae Kasei Co., Ltd. for technical advice. We also thank Dr. Kate Edmondson and Dr. Patrick Hughes of Bioedit, Ltd. for their critical reading of the manuscript.

\section{REFERENCES}

[1] Chen, D.Y., Hsieh, T.Y., Hsieh, C.W., Lin, F.J. and Lan, J.L. (2007) Increased apoptosis of peripheral blood lymphocytes and its association with interleukin-18 in patients with active untreated adult-onset Still's disease. Arthritis \& Rheumatism, 57, 1530-1538. doi:10.1002/art.23088

[2] Kitamura, T., Kabuyama, Y., Kamataki, A., Homma, M.K., Kobayashi, H., et al. (2008) Enhancement of lymphocyte migration and cytokine production by ephrinB1 system in rheumatoid arthritis. American Journal of Physiology-Cell Physiology, 294, 189-196. doi:10.1152/ajpcell.00314.2007

[3] Attur, M., Belitskaya-Lévy, I., Oh, C., Krasnokutsky, S., Greenberg, J., et al. (2011) Increased interleukin-1 $\beta$ gene expression in peripheral blood leukocytes is associated with increased pain and predicts risk for progression of symptomatic knee osteoarthritis. Arthritis \& Rheumatism, 63, 1908-1917. doi:10.1002/art.30360

[4] Mellman, I., Coukos, G. and Dranoff, G. (2011) Cancer immunotherapy comes of age. Nature, 480, 480-489. doi:10.1038/nature 10673

[5] Curran, K.J., Pegram, H.J. and Brentjens, R.J. (2012) Chimeric antigen receptors for $\mathrm{T}$ cell immunotherapy: Current understanding and future direction. The Journal of Gene Medicine, 6, 405-415. doi:10.1002/jgm.2604
[6] Yu, M., Stott, S., Toner, M., Maheswaran, S. and Haber, D.A. (2011) Circulating tumor cells: Approaches to isolation and characterization. The Journal of Cell Biology, 192, 373-382. doi:10.1083/jcb.201010021

[7] Bøyum, A. (1968) Isolation of mononuclear cells and granulocytes from human blood (Paper IV). Scandinavian Journal of Clinical and Laboratory Investigation Supplement, 97, 77-89.

[8] Bøyum, A., Brincker Fjerdingstad, H., Martinsen, I., Lea, T. and Løvhaug, D. (2002) Separation of human lymphocytes from citrated blood by density gradient (NycoPrep) centrifugation: Monocyte depletion depending upon activation of membrane potassium channels. Scandinavian Journal of Immunology, 56, 76-84. doi:10.1046/j.1365-3083.2002.01102.x

[9] Tomana, M., Parton, A. and Barnes, D.W. (2008) An improved method for separation of leucocytes from peripheral blood of the little skate (Leucoraja erinacea). Fish and Shellfish Immunology, 25, 188-190. doi:10.1016/j.fsi.2007.12.006

[10] http://www.pathology.unibe.ch/Lehre/downloads/Ficoll.pdf

[11] Cotter, M.J., Norman, K.E., Hellewell, P.G. and Ridger, V.C. (2001) A novel method for isolation of neutrophils from murine blood using negative immunomagnetic separation. American Journal of Pathology, 159, 473-481. doi:10.1016/S0002-9440(10)61719-1

[12] Burger, D.E., Wang, L., Ban, L., Okubo, Y., Kühtreiber, W.M., et al. (2011) Novel automated blood separations validate whole cell biomarkers. PLoS One, 6, e22430. doi:10.1371/journal.pone.0022430

[13] Ward, D.M. (2011) Conventional apheresis therapies: A review. Journal of Clinical Apheresis, 26, 230-238. doi:10.1002/jca.20302

[14] http://www.asahi-kasei.co.jp/medical/en/list/

[15] Ghosh, S., Kaplan, G. and Panaccione, R. (2009) Granulocyte-monocyte apheresis for the treatment of ulcerative colitis-Is this the end of the road? Nature Clinical Practice Gastroenterology \& Hepatology, 6, 6-7. doi:10.1038/ncpgasthep 1299

[16] Adacolumn. http://www.adacolumn.com/

[17] Danese, S., Angelucci, E., Marconi, S., Repici, A. and Malesci, A. (2008) Oral beclomethasone: A review of its use in inflammatory bowel disease. Mini Reviews in Medicine Chemistry, 8, 1459-1463. doi:10.2174/138955708786786444

[18] http://www.pall.com/medical_39479.asp

[19] Dzik, S. (1993) Leukodepletion blood filters: Filter design and mechanisms of leukocyte removal. Transfusion Medicine Reviews, 7, 65-77. doi:10.1016/S0887-7963(93)70125-X

[20] Ferrer, F., Rivera, J., Corral, J., González-Conejero, R. and Vicente, V. (2000) Evaluation of leukocyte-depleted platelet concentrates obtained by in-line filtration. Vox Sanguinis, 78, 235-241. doi:10.1046/j.1423-0410.2000.7840235.x

[21] Mascaretti, L., Baggi, L., Riva, M., Proserpio, P., Dassi, M., et al. (2002) Lymphocyte subsets in inline filtered packed red blood cell units: Comparison between low and 
high spin procedures. Transfusion and Apheresis Science, 26, 167-174. doi:10.1016/S1473-0502(02)00009-5

[22] Okuzaki, D., Kimura, S., Yabuta, N., Ohmine, T. and Nojima, H. (2011) LeukoCatch, a quick and efficient tool for the preparation of leukocyte extracts from blood.
BMC Clinical Pathology, 11, 9. doi:10.1186/1472-6890-11-9

[23] http://www.cancerimmunity.org/peptidedatabase/Tcellepi topes.htm

\section{LIST OF ABBREVIATIONS}

CTC: circulating tumor cells; CTC: cytotoxic T lymphocyte; LEK: lymphokine-activated killer cell, LY: lymphocyte; MO: monocyte; NE: neutrophil; NT: nontreated, PB: peripheral blood; PBL: peripheral blood leukocyte; PBMC: peripheral blood mononuclear cells; PBS: phosphate-buffered saline; PS: pass through; PTL: platelet; RBC: red blood cell.

\section{SUPPORTING INFORMATION}
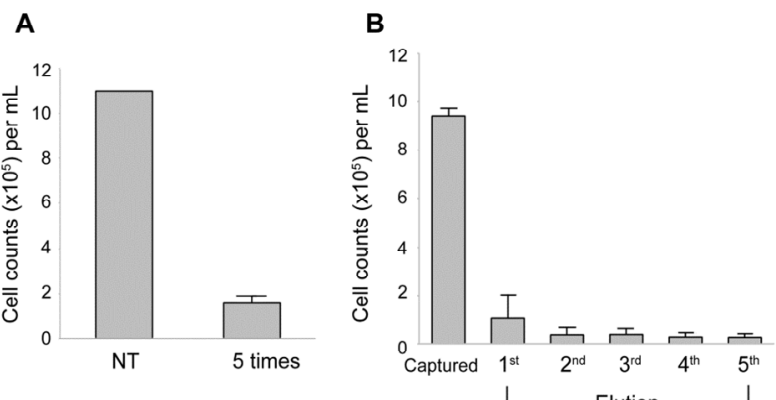

Figure S1. tLeukoCatch efficiently captures TRAMP-C1 cells. (A) TRAMP-C1 cells were passed through a tLeukoCatch five times. Bar graphs show the number of TRAMP-C1 cells before tLeukoCatch treatment (NT, nontreated) and after five passages through tLeukoCatch filters; (B) TRAMP-C1 cells were passed through tLeukoCatch five times. The captured TRAMP-C1 cells were eluted five times $\left(1^{\text {st }}\right.$ to $\left.5^{\text {th }}\right)$ with fresh DMEM. 